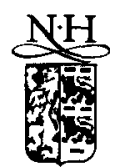

ELSEVIER

\title{
BRS symmetry versus supersymmetry in Yang-Mills-Chern-Simons theory
}

\author{
F. Ruiz Ruiz ${ }^{\mathrm{a}, \mathrm{b}, 1}$, P. van Nieuwenhuizen ${ }^{\mathrm{c}, 2}$ \\ a Institut für Theoretische Physik, Universität Heidelberg, Philosophenweg 16, 69120 Heidelberg, Germany \\ b NIKHEF, Postbus 41882, 1009 DB Amsterdam, The Netherlands \\ c Institute for Theoretical Physics, State University of New York at Stony Brook, Stony Brook, \\ NY 11794-3840, USA
}

Received 19 August 1996; accepted 22 November 1996

\begin{abstract}
We prove that three-dimensional $N=1$ supersymmetric Yang-Mills-Chern-Simons theory is finite to all loop orders. In general this leaves open the possibility that different regularization methods lead to different finite effective actions. We show that in this model dimensional regularization and regularization by dimensional reduction yield the same effective action. Consequently, the superfield approach preserves BRS invariance for this model.
\end{abstract}

PACS: 11.15.-q; 11.30.Pb; 11.10.Gh

Keywords: Dimensional regularization; Dimensional reduction; Supersymmetry; BRS invariance

\section{Introduction and conclusions}

One of the major unsolved problems in supersymmetry is the supersymmetric regularization of gauge theories. The renormalized effective action that results from using a particular regularization method and subtraction prescription can be made to satisfy either the supersymmetry or the gauge Ward identities by adding suitable finite local counterterms, but then the question arises whether the new renormalized effective action satisfies the Ward identities of the other symmetry. On algebraic cohomological grounds,

\footnotetext{
${ }^{1}$ E-mail: ruiz@thphys.uni-heidelberg.de. Alexander von Humboldt Research Fellow. Research partially supported by CICyT grant AEN95-1284E.

${ }^{2}$ E-mail: vannieu@insti.physics.sunysb.edu. Research supported by NSF grant Phy 9309888.
} 
it has been argued that for certain supersymmetric theories there exist renormalized effective actions which satisfy both sets of identities [1]. However, this does not tell us how to actually compute such preferred renormalized actions. In fact, no regularization method for four-dimensional supersymmetric gauge theories preserving both gauge invariance and supersymmetry is known to date.

In this article we study the formulation of a supersymmetric and gauge invariant regularization method in three dimensions. We consider $N=1$ supersymmetric YangMills-Chern-Simons theory, whose classical action

$$
S=\frac{1}{m} S_{\mathrm{YM}}+S_{\mathrm{CS}}
$$

consists of the sum of the Yang-Mills and the Chern-Simons actions, and study two regularization methods, ordinary dimensional regularization (or DReG) and regularization by dimensional reduction (or $\mathrm{DReD}$ ). We are interested in the difference $\Delta \Gamma=$ $\Gamma^{\mathrm{DReG}}\left[\psi, K_{\phi}\right]-\Gamma^{\mathrm{DReD}}\left[\psi, K_{\phi}\right]$ of the corresponding effective actions, where $\psi$ stands for all the fields and $K_{\phi}$ for the sources of the fields with non-linear BRS transformations. Since we will show that the theory is finite, the regularized effective actions $\Gamma^{\mathrm{DReG}}$ and $\Gamma^{\mathrm{DReD}}$ are also renormalized effective actions and $\Delta \Gamma$ is the difference of two renormalized effective actions. The first regularization method, namely DReG [2], preserves at all stages the BRS identities corresponding to local gauge invariance. This is so since, by treating the $\epsilon$-symbol $\epsilon^{\mu \nu \rho}$ in the classical Chern-Simons action as purely three dimensional [3], the kinetic matrix for the gauge field has an inverse in $d \geqslant 3$ dimensions and, by using this inverse as gauge propagator, the BRS symmetry is maintained in $d$ dimensions. Unfortunately, because for $d \neq 3$ the numbers of bosons and fermions are not equal, even when the Dirac algebra of the Feynman diagrams is performed in $d$ dimensions, DReG does not preserve supersymmetry manifestly. The second method, regularization by $\mathrm{DReD}$ [4], performs the algebra of all Feynman diagrams in terms of superfields and, only at the end, continues the momentum integrals to $d<3$ dimensions. The propagator that $\mathrm{DReD}$ uses for the gauge field is constructed with the $d$-dimensional $\left(d<3\right.$ ) momentum $p^{\mu}$ and the three-dimensional $\epsilon^{\mu \nu \rho}$ and $g_{\mu \nu}$, and is the inverse of the kinetic term in $d<3$ dimensions. However, due to the fact that DReD only continues the momenta to $d<3$, whereas the first $d$ components of the gauge field transform under BRS transformations in the same way as in the unregularized theory, the last $3-d$ components transform differently. For this reason, DReD does not preserve BRS invariance at all stages and runs the risk of violating the BRS identities, although it preserves supersymmetry manifestly. The well-known inconsistency of $\mathrm{DReD}$ [5] does not occur for this model (see Section 3).

Our strategy will be:

(i) To show that the theory is finite to all loops. In fact, by power counting the theory is only superrenormalizable, since it contains divergences at the one-, two- and three-loop levels. Hence, to compute radiative corrections, regularization is needed. We will use dimensional regularization to prove finiteness.

(ii) To use this to prove that the difference $\Delta \Gamma$ is proportional to a supersymmetric 
polynomial of the fields and their derivatives with only one free parameter.

(iii) To compute this parameter and to show that it vanishes.

Since the difference $\Delta \Gamma$ vanishes, one may use superspace methods to compute loop corrections while preserving both BRS invariance and supersymmetry. Our results depend critically on the fact that we are in three dimensions, and we make no claims concerning four-dimensional theories.

We will prove finiteness at one loop by using properties of dimensionally regularized integrals. At two loops, finiteness follows from the observation that there are no one-loop subdivergences and that the two-loop effective action $\Gamma_{2}^{\mathrm{DReG}}$ satisfies the BRS identity

$$
\Theta \Gamma_{2}^{\mathrm{DReG}}+\left(\Gamma_{1}^{\mathrm{DReG}}, \Gamma_{1}^{\mathrm{DReG}}\right)=0,
$$

so that the divergent part satisfies $\Theta \Gamma_{2 \text {,div }}^{\mathrm{DReG}}=0$, where $\Theta$ is the Slavnov-Taylor operator. Since the divergences in a 1PI Green function at $k$ loops are polynomials in the external momenta with degree equal to or less than the superficial overall UV degree of divergence $\bar{\omega}_{k}$ of the corresponding proper graphs, the most general form of $\Gamma_{2 \text {,div }}^{\mathrm{DReG}}$ is $\Gamma_{2 \text {,div }}^{\text {DReG }}=\frac{1}{d-3} P_{\bar{\omega}_{2}}\left[\partial, \psi, K_{\phi}\right]$, with $P_{\bar{\omega}_{2}}$ a certain polynomial in the fields, sources and their derivatives. To determine which terms are possible in $P_{\bar{\omega}_{2}}$, we need power counting for the various 1PI diagrams. We will find that no 1PI diagrams with BRS sources are superficially divergent, but only 1PI diagrams with fields. At one loop we will find quadratic, linear and logarithmic divergences, while at two loops only linear and logarithmic, and at three loops only logarithmic divergences will remain. In particular, $P_{\bar{\omega}_{2}}$ will only depend on the component fields in the gauge multiplet. We will show that no BRS invariant can be constructed out of these components, so that $\Gamma_{2 \text {,div }}^{\mathrm{DReG}}=0$. The same arguments will prove that the theory is also finite at three and higher loops.

There is a general theorem in quantum field theory [6,7] that states that if two different renormalization (not regularization) schemes yield the same Green functions up to $k-1$ loops, then at $k$ loops they give Green functions that can differ at most by a local finite polynomial in the external momenta of degree equal to the superficial overall UV degree of divergence $\bar{\omega}_{k}$ at $k$ loops. To go from the regularized to the renormalized Green function at $k$ loops, one must in general subtract the $k$-loop divergences. Given that in our case the theory is finite, and provided the regularized DReG and DReD expressions for the Green functions are identical at $k-1$ loops, it follows that at $k$ loops they can differ at most by a local finite polynomial in the external momenta. Using properties of dimensionally regularized integrals, we will show that DReG and DReD give the same expressions for all Green functions at one loop, so $\Delta \Gamma_{1}=0$. Then, the difference $\Delta \Gamma_{2}$ at two loops will be

$$
\Gamma_{2}^{\mathrm{DReG}}-\Gamma_{2}^{\mathrm{DReD}}=P_{\bar{\omega}_{2}}\left[\partial, \psi, K_{\phi}\right],
$$

with the same polynomial $P_{\bar{\omega}_{2}}$ that comes out in the analysis of finiteness. We already know that $\Gamma_{2}^{\mathrm{DReG}}$ satisfies the BRS identity (1.1). As for $\Gamma_{2}^{\mathrm{DReD}}$, since DReD manifestly preserves supersymmetry, it satisfies the supersymmetry Ward identity

$$
\delta \Gamma_{2}^{\mathrm{DReD}}=0
$$


where $\delta$ is the supersymmetry generator. Acting with $\delta$ on Eq. (1.1), using Eqs. (1.2) and (1.3), noting that $[\Theta, \delta]=0$ and recalling that $\Delta \Gamma_{1}=0$, leads to $\Theta \delta P_{\bar{\omega}_{2}}=0$

$$
0=\delta \Theta\left(\Gamma_{2}^{\mathrm{DReD}}+P_{\bar{\omega}_{2}}\right)+\delta\left(\Gamma_{1}^{\mathrm{DReD}}, \Gamma_{1}^{\mathrm{DReD}}\right)=\Theta \delta P_{\bar{\omega}_{2}} .
$$

As already mentioned, power counting implies that the terms allowed in $P_{\bar{\omega}_{2}}$ depend only on the component fields of the gauge multiplet. Hence, $\delta P_{\bar{\omega}_{2}}$ is a finite local functional of such fields. We will show (i) that it is not possible to construct a BRS invariant from these ingredients, so that $\delta P_{\bar{\omega}_{2}}=0$, and (ii) that the only supersymmetric invariant one can construct from $P_{\bar{\omega}_{2}}$ is

$$
P_{\bar{\omega}_{2}}^{\text {susy }}=\alpha m \int d^{3} x d^{2} \theta \Gamma^{a \alpha} \Gamma_{\alpha}^{a},
$$

with $\Gamma_{\alpha}^{a}$ the basic spinor superfield containing the gauge multiplet and $\alpha$ a constant. Finally, we compute $\alpha$ and obtain zero. The same arguments as at two loops imply that the difference $\Delta \Gamma$ also vanishes at three and higher loops, except that in this case there is not even a derivative in $P_{\bar{\omega}_{3}}$ so that no supersymmetric invariant can be constructed. Hence, $P_{\bar{\omega}_{3}}=0$.

The paper is organized as follows. In Section 2 we introduce our notation and write the classical action and the BRS and supersymmetry transformations in superfields and components. We need the component expressions since ordinary DReG can not be formulated in terms of superfields. To have a supersymmetric gauge-fixed classical action we will work in the supersymmetric Landau gauge, which although linearly realized in terms of the superfield $\Gamma_{\alpha}^{a}$ will give non-linear gauge conditions for the components of the gauge multiplet. In Section 3 we define DReG and explain how to handle the $\epsilon^{\mu \nu \rho}$ in the classical action. We also briefly recall some elements of DReD. Section 4 contains our proof of perturbative finiteness, and Section 5 the proof that DReG and DReD yield the same expression for the effective action. Section 6 contains some further remarks.

\section{Classical action, gauge-fixing and power counting}

We will work in Minkowski space-time with metric $g_{\mu \nu}=\operatorname{diag}(-,+,+)$ and completely antisymmetric tensor $\epsilon^{\mu \nu \rho}$ defined by $\epsilon^{012}=1$. We recall that in three dimensions the Lorentz algebra can be realized as $s o(1,2)$ or as $\operatorname{sl}(2, \mathbb{R})$, and that the fundamental representation has dimension two and acts on real Majorana spinors $\psi^{\alpha}$. Spinor indices will be denoted by Greek letters $\alpha, \beta, \ldots$ and will be raised and lowered with the rank-two antisymmetric tensors $\epsilon^{\alpha \beta}$ and $\epsilon_{\alpha \beta}$ and the northwest-southeast convention. That is, $\psi^{\alpha}=\epsilon^{\alpha \beta} \psi_{\beta}$ and $\psi_{\alpha}=\psi^{\beta} \epsilon_{\beta \alpha}$. We define $\epsilon_{\alpha \beta}$ by $\epsilon_{12}=1$, which together with our contraction convention implies that $\epsilon^{12}=1$. In this paper we will consider $N=1$ supersymmetry, so all our spinors will be Majorana. The vector representation of the Lorentz group has dimension three and acts on real vectors. A vector $v$ admits an $s o(1,2)$ realization as a space-time vector $v^{\mu}$ and an $s l(2, \mathbb{R})$ realization as a symmetric rank-two tensor $v^{\alpha}{ }_{\beta}$. To go from one to the other we use the Dirac gamma matrices $\gamma_{\mu}$ : 


$$
v_{\beta}^{\alpha}=\left(\gamma_{\mu}\right)_{\beta}^{\alpha} v^{\mu} .
$$

The Dirac gamma matrices $\gamma^{\mu}$ satisfy $\left\{\gamma^{\mu}, \gamma^{\nu}\right\}=2 g^{\mu \nu}$ and have spinor indices $\left(\gamma^{\mu}\right)^{\alpha}{ }_{\beta}$. When necessary, we will use the real representation $\gamma^{0}=-i \sigma^{2}, \gamma^{1}=\sigma^{1}, \gamma^{2}=\sigma^{3}$. The charge conjugate of a spinor $\psi^{\alpha}$ is defined by $\bar{\psi}_{\beta}=\psi^{\alpha} C_{\alpha \beta}$, with $C_{\alpha \beta}=-i \epsilon_{\alpha \beta}$ the charge conjugation matrix. By definition, $C$ satisfies $C=-C^{T}$ and $\left(C \gamma^{\mu}\right)^{T}=C \gamma^{\mu}$. Finally, we recall that in three dimensions the matrices $\left\{1, \gamma_{\mu}\right\}$ form a basis of the Clifford algebra and that given four spinors $\psi_{i}(i=1,2,3,4)$ the Fierz identity takes the form

$$
\left(\bar{\psi}_{1} M \psi_{2}\right)\left(\bar{\psi}_{3} N \psi_{4}\right)=-\frac{1}{2}\left[\left(\bar{\psi}_{1} M N \psi_{4}\right)\left(\bar{\psi}_{3} \psi_{2}\right)+\left(\bar{\psi}_{1} M \gamma^{\mu} N \psi_{4}\right)\left(\bar{\psi}_{3} \gamma_{\mu} \psi_{2}\right)\right] .
$$

Our conventions for superfields are as follows. Superspace is parameterized by three real space-time coordinates $x^{\alpha \beta}$ and two real anticommuting Majorana spinor coordinates $\theta^{\alpha}$. Space-time derivatives are denoted by $\partial_{\alpha \beta} \equiv\left(\gamma^{\mu}\right)_{\alpha \beta} \partial_{\mu}$, ordinary spinor derivatives by $\partial_{\alpha}$ and spinor superderivatives by $D_{\alpha}=\partial_{\alpha}+i \theta^{\beta} \partial_{\beta \alpha}$. Useful identities to project onto components are $\left\{D_{\alpha}, D_{\beta}\right\}=2 i \partial_{\alpha \beta},\left[D_{\alpha}, D_{\beta}\right]=-\epsilon_{\alpha \beta} D^{2}$ and $D^{\beta} D_{\alpha} D_{\beta}=0$. From $\left\{\partial_{\alpha}, \theta^{\beta}\right\}=\delta_{\alpha}{ }^{\beta}$ and $\left[\partial_{\alpha \beta}, x^{\gamma \delta}\right]=-\frac{1}{2}\left(\delta_{\alpha}^{\gamma} \delta_{\beta}{ }^{\delta}+\delta_{\alpha}{ }^{\delta} \delta_{\beta}^{\gamma}\right)$, it follows that $\partial_{\alpha}$ is real and that $\partial_{\alpha \beta}$ is imaginary, so that $D_{\alpha}$ is real. As for the measure in superspace, we note that $d^{3} x$ is real and $d^{2} \theta \equiv-2 d \theta^{1} d \theta^{2}=D^{2}$ imaginary. Under a supersymmetry transformation,

$$
\delta x^{\alpha \beta}=a^{\alpha \beta}-2 i \varepsilon^{(\alpha} \theta^{\beta)}, \quad \delta \theta^{a}=\varepsilon^{\alpha},
$$

where $a^{\alpha \beta}$ is a real commuting constant, $\varepsilon^{(\alpha} \theta^{\beta)}=\frac{1}{2}\left(\varepsilon^{\alpha} \theta^{\beta}+\varepsilon^{\beta} \theta^{\alpha}\right)$, and $\epsilon^{\alpha}$ is an anticommuting constant Majorana spinor. The supercharge $Q_{\alpha}$ is $Q_{\alpha}=\partial_{\alpha}-i \theta^{\beta} \partial_{\beta \alpha}$. As usual, a superfield $\Psi(x, \theta)$ transforms linearly under the action of the supercharge: $\delta \Psi=\epsilon^{\alpha} Q_{\alpha} \Psi$.

\subsection{Superfield classical action}

We assume the gauge algebra to be a real, compact, semi-simple Lie algebra, so that the structure constants $f^{a b c}$ can be taken completely anti-symmetric without loss of generality. The real gauge field $A_{\alpha \beta}^{a}$ is part of a vector supermultiplet described by a Majorana spinor gauge potential $\Gamma_{\alpha}^{a}$ [8]. Besides $A_{\alpha \beta}^{a}$, the supermultiplet contains a real scalar field $H^{a}$ and two anticommuting Majorana spinors $\chi_{\alpha}^{a}$ and $\lambda_{\alpha}^{a}$. The superfield $\Gamma_{\alpha}^{a}$ defines a real vector gauge potential $\Gamma_{\alpha \beta}^{a}$ and an imaginary spinor field strength $W_{\alpha}^{a}$ through the expressions

$$
\begin{aligned}
\Gamma_{\alpha \beta}^{a} & =D_{(\alpha} \Gamma_{\beta)}^{a}+\frac{i}{2} f^{a b c} \Gamma_{\alpha}^{b} \Gamma_{\beta}^{c}, \\
W_{\alpha}^{a} & =D^{\beta} D_{\alpha} \Gamma_{\beta}^{a}+i f^{a b c} \Gamma^{b \beta} D_{\beta} \Gamma_{\alpha}^{c}-\frac{1}{3} f^{a b c} f^{c d e} \Gamma^{b \beta} \Gamma_{\beta}^{d} \Gamma_{\alpha}^{e} .
\end{aligned}
$$

The reality of $\Gamma_{\alpha \beta}^{a}$ follows from writing $D_{\alpha} \Gamma_{\beta}^{a}$ as $\left\{D_{\alpha}, \Gamma_{\beta}^{a}\right\}$ and using that $D_{\alpha}$ is real. As a check on the coefficients in $W_{\alpha}^{a}$, one may verify that the Bianchi identity $\nabla^{\alpha} W_{\alpha}^{a}=0$ 
Table 1

Mass dimension, ghost number and Grassmann grading ( $\mathrm{A}=$ anticommuting, $\mathrm{C}=$ commuting $)$ of component fields

\begin{tabular}{llllllllllllllll}
\hline Field & $\chi_{\alpha}^{a}$ & $H^{a}$ & $A_{\alpha \beta}^{a}$ & $\lambda_{\alpha}^{a}$ & $b^{a}$ & $\zeta_{\alpha}^{a}$ & $h^{a}$ & $c^{a}$ & $\varphi_{\alpha}^{a}$ & $\omega^{a}$ & $\hat{c}^{a}$ & $\hat{\varphi}_{\alpha}^{a}$ & $\hat{\omega}^{a}$ \\
\hline Mass dim. & $1 / 2$ & 1 & 1 & $3 / 2$ & 1 & $3 / 2$ & 2 & $1 / 2$ & 1 & $3 / 2$ & $1 / 2$ & 1 & $3 / 2$ \\
Ghost no. & 0 & 0 & 0 & 0 & 0 & 0 & 0 & 1 & 1 & 1 & -1 & -1 & -1 \\
Grassmann grad. & $\mathrm{A}$ & $\mathrm{C}$ & $\mathrm{C}$ & $\mathrm{A}$ & $\mathrm{C}$ & $\mathrm{A}$ & $\mathrm{C}$ & $\mathrm{A}$ & $\mathrm{C}$ & $\mathrm{A}$ & $\mathrm{A}$ & $\mathrm{C}$ & $\mathrm{A}$ \\
\hline
\end{tabular}

is satisfied. In terms of $\Gamma_{\alpha}^{a}, \Gamma_{\alpha \beta}^{a}$ and $W_{\alpha}^{a}$, the components of the gauge multiplet are given by

$$
\chi_{\alpha}^{a}=\Gamma_{\alpha}^{a}\left|, \quad H^{a}=\frac{1}{2} D^{\alpha} \Gamma_{\alpha}^{a}\right|, \quad A_{\alpha \beta}^{a}=\Gamma_{\alpha \beta}^{a}\left|, \quad \lambda_{\alpha}^{a}=-\frac{i}{2} W_{\alpha}^{a}\right|,
$$

where the vertical bar denotes projection onto $\theta^{\alpha}=0$ and the numerical factors have been adjusted so that the Yang-Mills and Chern-Simons component actions have the standard form [see Eqs. (2.12)-(2.13)]. We take $\Gamma_{\alpha}^{a}$ to have mass dimension 1/2, so that using that $\theta^{\alpha}$ has mass dimension $-1 / 2$, the components have mass dimensions as in Table 1.

The classical $N=1$ Yang-Mills-Chern-Simons action has the form [8]

$$
S=\frac{1}{m} S_{\mathrm{YM}}+S_{\mathrm{CS}},
$$

where

$$
\begin{aligned}
S_{\mathrm{YM}}= & -\frac{1}{32 g^{2}} \int d^{3} x d^{2} \theta W^{a \alpha} W_{\alpha}^{a}, \\
S_{\mathrm{CS}}= & \frac{i}{16 g^{2}} \int d^{3} x d^{2} \theta\left[\left(D^{\alpha} \Gamma^{a \beta}\right)\left(D_{\beta} \Gamma_{\alpha}^{a}\right)+\frac{2 i}{3} f^{a b c} \Gamma^{a \alpha} \Gamma^{b \beta}\left(D_{\beta} \Gamma_{\alpha}^{c}\right)\right. \\
& \left.-\frac{1}{6} f^{a b c} f^{c d e} \Gamma^{a \alpha} \Gamma^{b \beta} \Gamma_{\alpha}^{d} \Gamma_{\beta}^{e}\right]
\end{aligned}
$$

are the Yang-Mills and Chern-Simons actions, $m$ is a parameter with dimensions of mass and $g$ is a dimensionless coupling constant. Both $S_{\mathrm{YM}}$ and $S_{\mathrm{CS}}$ are invariant under gauge transformations $\delta_{\Omega} \Gamma_{\alpha}^{a}=\left(\nabla_{\alpha} \Omega\right)^{a}$, where $\nabla_{\alpha}^{a b}=\delta^{a b} D_{\alpha}+i f^{a c b} \Gamma_{\alpha}^{c}$ is the spinor covariant derivative and $\Omega^{a}$ is an arbitrary real scalar superfield, and under supersymmetry transformations $\delta \Gamma_{\alpha}^{a}=\varepsilon^{\beta} Q_{\beta} \Gamma_{\alpha}^{a}$. To fix the gauge, we impose the supersymmetric Landau condition

$$
D^{\alpha} \Gamma_{\alpha}^{a}=0
$$

The Faddeev-Popov procedure then adds to the classical action a contribution

$$
S_{\mathrm{GF}}=\frac{i}{4} \int d^{3} x d^{2} \theta\left[B^{a}\left(D^{\alpha} \Gamma_{\alpha}^{a}\right)+i \hat{C}^{a}\left(D^{\alpha} \nabla_{\alpha} C\right)^{a}\right]
$$

with $B^{a}$ a real commuting Lagrange multiplier superfield of mass dimension 1 imposing the condition $D^{\alpha} \Gamma_{\alpha}^{a}=0$, and $\hat{C}^{a}$ and $C^{a}$ real anticommuting antighost and ghost 
Table 2

Mass dimension, ghost number and Grassmann grading of external sources

\begin{tabular}{llllllll}
\hline External source & $\kappa_{\alpha}^{a}$ & $G^{a}$ & $K_{\alpha \beta}^{a}$ & $\sigma_{\alpha}^{a}$ & $\ell^{a}$ & $\tau_{\alpha}^{a}$ & \multicolumn{1}{l}{$L^{a}$} \\
\hline Mass dim. & 1 & $3 / 2$ & $3 / 2$ & 2 & 1 & $3 / 2$ & 2 \\
Ghost no. & -1 & -1 & -1 & -1 & -2 & -2 & -2 \\
Grassmann grad. & $\mathrm{C}$ & $\mathrm{A}$ & $\mathrm{A}$ & $\mathrm{C}$ & $\mathrm{C}$ & $\mathrm{A}$ & $\mathrm{C}$ \\
\hline
\end{tabular}

superfields of mass dimension $1 / 2$. After gauge fixing, gauge invariance is replaced by BRS invariance. To obtain the BRS variation, we replace the gauge parameter $\Omega^{a}$ with $-i \eta C^{a}$ and $\delta_{\Omega}$ with $\eta s$, where $\eta$ is a Grassmann constant, $C^{a}$ is the ghost superfield and $s$ is the BRS operator. This and the requirement of nilpotency for $s$ gives the following BRS transformations:

$$
s \Gamma_{\alpha}^{a}=i\left(\nabla_{\alpha} C\right)^{a}, \quad s B^{a}=0, \quad s \hat{C}^{a}=B^{a}, \quad s C^{a}=-\frac{1}{2} f^{a b c} C^{b} C^{c} .
$$

It can easily be checked that $s$ leaves $S_{\mathrm{YM}}, S_{\mathrm{CS}}$ and $S_{\mathrm{GF}}$ invariant. We note that $[s, \delta]=0$ because they act in different spaces. We define the field components of $B^{a}, C^{a}$ and $\hat{C}^{a}$ through the projections (see Table 1 for their mass dimension and Grassmann grading)

$$
\begin{aligned}
& b^{a}=B^{a}\left|, \quad c^{a}=C^{a}, \quad \hat{c}^{a}=\hat{C}^{a}\right|, \\
& \zeta_{\alpha}^{a}=i D_{\alpha} B^{a}\left|, \quad \varphi_{\alpha}^{a}=D_{\alpha} C^{a}\right|, \quad \hat{\varphi}_{\alpha}^{a}=D_{\alpha} \hat{C}^{a} \mid, \\
& h^{a}=-\frac{i}{2} D^{2} B^{a}\left|, \quad \omega^{a}=-\frac{i}{2} D^{2} C^{a}\right|, \quad \hat{\omega}^{a}=-\frac{i}{2} D^{2} \hat{C}^{a} \mid .
\end{aligned}
$$

With the purpose of studying BRS invariance at the quantum level, we introduce commuting external supersources $K_{\Gamma}^{a \alpha}$ and $K_{C}^{a}$ coupled to the non-linear BRS transforms $s \Gamma_{\alpha}^{a}$ and $s C^{a}$ :

$$
S_{\mathrm{ES}}=\frac{i}{2} \int d^{3} x d^{2} \theta\left(\frac{1}{2} K_{\Gamma}^{a \alpha} s \Gamma_{\alpha}^{a}-K_{C}^{a} s C^{a}\right) .
$$

We define the components of $K_{\Gamma}^{a \alpha}$ and $K_{C}^{a}$ by the projections

$$
\begin{aligned}
\kappa_{\alpha}^{a} & =K_{\alpha \Gamma}^{a} \mid, & \ell^{a} & =K_{C}^{a} \mid, \\
G^{a} & =-\frac{i}{2} D^{\alpha} K_{\alpha \Gamma}^{a} \mid, & \tau_{\alpha}^{a} & =i D_{\alpha} K_{C}^{a} \mid, \\
K_{\alpha \beta}^{a} & =i D_{(\alpha} K_{\beta) \Gamma}^{a} \mid, & L^{a} & =-\frac{i}{2} D^{2} K_{C}^{a} \mid, \\
\sigma_{\alpha}^{a} & =-\frac{i}{2} D^{\beta} D_{\alpha} K_{\beta \Gamma}^{a} \mid, & &
\end{aligned}
$$

from which the mass dimensions and Grassmann gradings in Table 2 follow.

All in all, we take as starting point the tree-level action

$$
\Gamma_{0}=\frac{1}{m} S_{\mathrm{YM}}+S_{\mathrm{CS}}+S_{\mathrm{GF}}+S_{\mathrm{ES}} \text {. }
$$


Table 3

IPI superficially divergent super-diagrams for $\Gamma_{0}$

\begin{tabular}{lll}
\hline External lines & 1 loop & 2 loops \\
\hline$r^{2}$ & $\bar{\omega}=1$ & $\bar{\omega}=0$ \\
$r^{3} \quad r^{4}$ & $\bar{\omega}=0$ & \\
\hline
\end{tabular}

Superpower counting for $\Gamma_{0}$ gives a finite number of superficially divergent 1PI diagrams, namely those in Table 3 , where $\bar{\omega}$ denotes the overall superficial UV degree of divergence. This shows that the theory is superrenormalizable. We remark that there are no superficially divergent diagrams with either ghosts $C^{a}, \hat{C}^{a}$ or sources $K_{\Gamma}^{a \alpha}, K_{C}^{a}$ as external lines. The connected generating functional $W\left[J_{\Psi}, K_{\Phi}\right]$ is given by

$$
\exp \left\{i W\left[J_{\Psi}, K_{\Phi}\right]\right\}=\int \prod_{\Psi}[d \Psi] \exp \left\{i\left(\Gamma_{0}\left[K_{\phi}\right]+\int d^{3} x d^{2} \theta J_{\Psi} \Psi\right)\right\}
$$

where we have introduced external sources $J_{\psi}^{a}=J_{\Gamma}^{a \alpha}, J_{B}^{a}, J_{C}^{a}, J_{\hat{C}}^{a}$ for the fields $\Psi^{a}=$ $\Gamma_{\alpha}^{a}, B^{a}, \hat{C}^{a}, C^{a}$ and used the notation $K_{\phi}^{a}=K_{\Gamma}^{\alpha \alpha}, K_{C}^{a}$ for the sources coupled to the non-linear BRS transforms. Performing a BRS change of variables under the integral, using that there are no BRS anomalies ${ }^{3}$ and defining the effective action $\Gamma\left[\Psi, K_{\phi}\right]$ as the Legendre transform [9] of $W\left[J_{\psi}, K_{\Phi}\right]$, we find the BRS identity

$$
\int d^{3} x d^{2} \theta\left(\frac{\delta \Gamma}{\delta \Gamma_{\alpha}^{a}} \frac{\delta \Gamma}{\delta K_{\Gamma}^{a \alpha}}+\frac{\delta \Gamma}{\delta C^{a}} \frac{\delta \Gamma}{\delta K_{C}^{a}}+B^{a} \frac{\delta \Gamma}{\delta \hat{C}^{a}}\right)=0
$$

To regularize and renormalize the theory, one can think of using regularization by DReD [4]. This keeps the advantages of the superfield formalism. However, there is no a priori reason why the resulting effective action should satisfy the BRS identity $(2.11)$, since DReD is not manifestly BRS invariant.

\subsection{Component classical action}

To use ordinary DReG, we turn now to the component formalism. Using Eqs. (2.1), (2.7) and (2.9), it is not difficult to see that in terms of component fields $S_{\mathrm{YM}}, S_{\mathrm{CS}}, S_{\mathrm{GF}}$ and $S_{\mathrm{ES}}$ take the form

$$
\begin{aligned}
& S_{\mathrm{YM}}=\frac{1}{g^{2}} \int d^{3} x\left[-\frac{1}{4} F_{\mu \nu}^{a} F^{a \mu \nu}-\frac{1}{2} \bar{\lambda}^{a}(\not D \lambda)^{a}\right], \\
& S_{\mathrm{CS}}=\frac{1}{g^{2}} \int d^{3} x\left[\epsilon^{\mu \nu \rho}\left(\frac{1}{2} A_{\mu}^{a} \partial_{\nu} A_{\rho}^{a}+\frac{1}{6} f^{a b c} A_{\mu}^{a} A_{\nu}^{b} A_{\rho}^{c}\right)-\frac{1}{2} \bar{\lambda}^{a} \lambda^{a}\right], \\
& S_{\mathrm{GF}}=\int d^{3} x\left\{-b^{a} \partial_{\mu} V^{a \mu}-\left(\partial^{\mu} \hat{c}^{a}\right)\left(\partial_{\mu} c^{a}+f^{a b c} V_{\mu}^{b} c^{c}-\frac{i}{2} f^{a b c} \bar{\chi}^{b} \gamma_{\mu} \varphi^{c}\right)\right.
\end{aligned}
$$

\footnotetext{
${ }^{3}$ Absence of BRS anomalies follows from the fact that DReG preserves BRS invariance in $n$ dimensions for our model (see Section 3). In four-dimensional chiral gauge theories, this is not the case.
} 


$$
\begin{aligned}
& -\bar{\zeta}^{a} \Lambda^{a}-\overline{\hat{\varphi}}^{a}\left[\not \varphi^{a}+f^{a b c}\left(i \Lambda^{b} c^{c}+\frac{i}{2} \gamma^{\mu} \chi^{b} \partial_{\mu} c^{c}+\frac{1}{2} y^{b} \varphi^{c}\right.\right. \\
& \left.\left.\left.-\frac{1}{2} H^{b} \varphi^{c}+\frac{i}{2} \chi^{b} \omega^{c}\right)\right]-h^{a} H^{a}+\hat{\omega}^{a}\left(\omega^{a}+f^{a b c} H^{b} c^{c}-\frac{i}{2} f^{a b c} \bar{\chi}^{b} \varphi^{c}\right)\right\},
\end{aligned}
$$

$$
S_{\mathrm{ES}}=\int d^{3} x\left[i \bar{\kappa}^{a} s \Lambda^{a}+K^{a \mu} s V_{\mu}^{a}+G^{a} s H^{a}+i \bar{\sigma}^{a} s \chi^{a}+\ell^{a} s \omega^{a}+i \bar{\tau}^{a} s \varphi^{a}+L^{a} s c^{a}\right]
$$

Here $F_{\mu \nu}^{a}=\partial_{\mu} A_{\nu}^{a}-\partial_{\nu} A_{\mu}^{a}+f^{a b c} A_{\mu}^{b} A_{\nu}^{c}$ and $D_{\mu}^{a b}=\delta^{a b} \partial_{\mu}+f^{a c b} A_{\mu}^{c}$ denote the field strength and the covariant derivative, and $V_{\mu}^{a}$ and $\Lambda^{a}$ are given by

$$
\begin{aligned}
& V_{\mu}^{a}=A_{\mu}^{a}+\frac{1}{4} f^{a b c} \bar{\chi}^{b} \gamma_{\mu} \chi^{c}, \\
& A^{a}=\lambda^{a}+\not \chi^{a}+\frac{1}{2} f^{a b c} A^{b} \chi^{c}-\frac{1}{2} f^{a b c} H^{b} \chi^{c}-\frac{1}{24} f^{a b c} f^{c d e} \gamma^{\mu} \chi^{b}\left(\bar{\chi}^{d} \gamma_{\mu} \chi^{e}\right)
\end{aligned}
$$

The fields $V_{\mu}^{a}$ and $A^{a}$ have a very simple expression as superfield projections:

$$
V_{\alpha \beta}^{a}=D_{(\alpha} \Gamma_{\beta)}^{a}\left|, \quad A_{\alpha}^{a}=\frac{i}{2} D_{\alpha} D^{\beta} \Gamma_{\beta}^{a}\right|
$$

The BRS and supersymmetry transformation laws for the components are obtained from the BRS and supersymmetry transformation laws for the superfields and the definition of components as projections. After some algebra, we obtain

$$
\begin{array}{rlrl}
\Gamma_{\alpha}^{a}: & s \chi^{a}=i \varphi^{a}-f^{a b c} \chi^{b} c^{c} & B^{a}: s b^{a} & =0 \\
s A_{\mu}^{a} & =\left(D_{\mu} c\right)^{a} & s \zeta^{a} & =0 \\
s H^{a} & =\omega^{a}+f^{a b c} H^{b} c^{c}-\frac{i}{2} f^{a b c} \bar{\chi}^{b} \varphi^{c} & s h^{a} & =0 \\
s \lambda^{a} & =-f^{a b c} \lambda^{b} c^{c} & & \\
\hat{C}^{a}: s \hat{c}^{a} & =b^{a} & C^{a}: s c^{a} & =-\frac{1}{2} f^{a b c} c^{b} c^{c} \\
s \hat{\varphi}^{a} & =i \zeta^{a} & s \varphi^{a} & =f^{a b c} \varphi^{b} c^{c} \\
s \hat{\omega}^{a} & =h^{a} & s \omega^{a} & =-f^{a b c} \omega^{b} c^{c}-\frac{1}{2} f^{a b c} \bar{\varphi}^{b} \varphi^{c}
\end{array}
$$

for the BRS transformations, and 


$$
\begin{aligned}
& \Gamma_{\alpha}^{a}: \delta \chi^{a}=\psi^{a} \varepsilon-H^{a} \varepsilon \\
& B^{a}: \delta b^{a}=-\bar{\zeta}^{a} \varepsilon \\
& \delta A_{\mu}^{a}=\bar{\varepsilon} \gamma_{\mu} \lambda^{a}+\bar{\varepsilon}\left(D_{\mu} \chi\right)^{a} \\
& \delta \zeta^{a}=h^{a} \varepsilon-\not b^{a} \varepsilon \\
& \delta H^{a}=-\bar{\varepsilon} \Lambda^{a} \\
& \delta \lambda^{a}=-\frac{1}{2} \gamma^{\mu} \gamma^{\nu} F_{\mu \nu}^{a} \varepsilon+f^{a b c} \lambda^{b}\left(\bar{\chi}^{c} \varepsilon\right) \\
& \delta h^{a}=\bar{\varepsilon} \not \zeta \zeta^{a} \\
& \hat{C}^{a}: \delta \hat{c}^{a}=i \overline{\hat{\varphi}}^{a} \varepsilon \\
& C^{a}: \delta c^{a}=i \bar{\varphi}^{a} \varepsilon \\
& \delta \hat{\varphi}^{a}=-i \not \partial \hat{c}^{a} \varepsilon+i \hat{\omega}^{a} \varepsilon \\
& \delta \varphi^{a}=-i \not c^{a} \varepsilon+i \omega^{a} \varepsilon \\
& \delta \hat{\omega}^{a}=i \vec{\varepsilon} \not \hat{\varphi}^{a} \\
& \delta \omega^{a}=i \bar{\varepsilon} \not \varphi^{a} \\
& K_{\Gamma}^{a \alpha}: \delta \kappa^{a}=i \not k^{a} \varepsilon+i G^{a} \varepsilon \\
& \delta G^{a}=i \bar{\varepsilon} \not \partial \kappa^{a}+i \bar{\varepsilon} \sigma^{a} \\
& K_{C}^{a}: \delta \ell^{a}=\bar{\varepsilon} \tau^{a} \\
& \delta K_{\mu}^{a}=i \bar{\varepsilon} \partial_{\mu} \kappa^{a}+i \bar{\varepsilon} \gamma_{\mu} \sigma^{a} \\
& \delta \tau^{a}=i \not \supset \ell^{a} \varepsilon-i L^{a} \varepsilon \\
& \delta \sigma^{a}=\frac{i}{2} \gamma^{\mu} \gamma^{\nu} \varepsilon\left(\partial_{\mu} K_{\nu}^{a}-\partial_{\nu} K_{\mu}^{a}\right) \\
& \delta L^{a}=\bar{\varepsilon} \not \partial \tau^{a}
\end{aligned}
$$

for the supersymmetry transformations. To understand the gauge (2.4) in terms of components, it is convenient to recast $S_{\mathrm{GF}}$ in $\mathrm{Eq}$. (2.5) as

$$
S_{\mathrm{GF}}=\int d^{3} x s\left(-\hat{c}^{a} \partial_{\mu} V^{a \mu}+i \overline{\hat{\varphi}}^{a} \Lambda^{a}-\hat{\omega}^{a} H^{a}\right) .
$$

It then becomes clear that $S_{\mathrm{GF}}$ imposes the conditions

$$
\partial_{\mu} V^{a \mu}=0, \quad A^{a}=0, \quad H^{a}=0
$$

through the Lagrange multipliers $b^{a}, \zeta^{a}, h^{a}$, and that associated with these conditions there are ghost-antighost pairs $\left(c^{a}, \hat{c}^{a}\right),\left(\varphi^{a}, \hat{\varphi}^{a}\right),\left(\omega^{a}, \hat{\omega}^{a}\right)$. As a check, one may verify that the conditions (2.18) are invariant under the component supersymmetry transformation laws given above. BRS and supersymmetry invariance for $S_{\mathrm{YM}}$ and $S_{\mathrm{CS}}$ written in components is straightforward to check. As regards $S_{\mathrm{GF}}$ and $S_{\mathrm{ES}}, \mathrm{BRS}$ invariance is trivial and supersymmetry invariance is easily verified if one uses $[s, \delta]=0$ and the supersymmetry transformations for $V_{\mu}^{a}$ and $A^{a}$ :

$$
\delta V_{\mu}^{a}=\bar{\varepsilon} \gamma_{\mu} A^{a}-\frac{1}{2} \bar{\varepsilon}\left(\gamma_{\mu} \gamma_{\nu}-\gamma_{\nu} \gamma_{\mu}\right) \partial^{\nu} \chi^{a}, \quad \delta A^{a}=\partial V^{a} \varepsilon-\not \partial H^{a} \varepsilon
$$

Introducing real external sources $J_{\psi}^{a}$ for the fields $\psi^{a}=\chi^{a}, V_{\mu}^{a}, H^{a}, \Lambda^{a}, b^{a}, \zeta^{a}, h^{a}$, $c^{a}, \varphi^{a}, \omega^{a}, \hat{c}^{a}, \hat{\varphi}^{a}, \hat{\omega}^{a}$, denoting by $K_{\phi}^{a}$ the external sources for the non-linear BRS transforms $s \phi^{a}\left(\phi^{a}=\chi^{a}, V_{\mu}^{a}, H^{a}, A^{a}, c^{a}, \varphi^{a}, \omega^{a}\right)$ and following Zinn-Justin [9], it is straightforward to see that the effective action $\Gamma \equiv \Gamma\left[\psi, K_{\phi}\right]$ generating 1PI Green functions of the fields $\psi^{a}$ and the sources $K_{\phi}^{a}$ satisfies the BRS identity

$$
\int d^{3} x\left(\sum_{\phi} \frac{\delta \Gamma}{\delta \phi} \frac{\delta \Gamma}{\delta K_{\phi}}+b \frac{\delta \Gamma}{\delta \hat{c}}+i \bar{\zeta} \frac{\delta \Gamma}{\delta \overline{\hat{\varphi}}}+h \frac{\delta \Gamma}{\delta \hat{\omega}}\right)=0 .
$$


We remark that $\Gamma$ generates 1PI Green functions for the fields $V_{\mu}^{a}$ and $A^{a}$ and not for the elementary fields $A_{\mu}^{a}$ and $\lambda^{a}$. This is due to the fact that $S_{\mathrm{ES}}$ in Eq. (2.15) introduces external sources for the BRS variations of $V_{\mu}^{a}$ and $A^{a}$, and not for those of $A_{\mu}^{a}$ and $\lambda^{a}$. To end up with a BRS identity for an effective action $\Gamma^{\prime}$ generating 1PI Green functions for the fields $A_{\mu}^{a}$ and $\lambda^{a}$, we must replace $S_{\mathrm{ES}}$ with

$$
S_{\mathrm{ES}}^{\prime}=\int d^{3} x\left[i \bar{\kappa}^{a} s \lambda^{a}+K^{a} s A^{a}+G^{a} s H^{a}+i \bar{\sigma}^{a} s \chi^{a}+\ell^{a} s \omega^{a}+i \bar{\tau}^{a} s \varphi^{a}+L^{a} s c^{a}\right]
$$

The problem is then that

$$
\Gamma_{0}^{\prime}=\frac{1}{m} S_{\mathrm{YM}}+S_{\mathrm{CS}}+S_{\mathrm{GF}}+S_{\mathrm{ES}}^{\prime}
$$

is not what results from projecting onto components the classical action $\Gamma_{0}$ written in terms of superfields, so we will not be concerned with the effective action $\Gamma^{\prime}$ built upon $\Gamma_{0}^{\prime}$. Coming back to the BRS identity (2.19) and the effective action $\Gamma$, we will need later the explicit form of this identity at one, two and three loops. To obtain it, we write for $\Gamma$ a loop expansion

$$
\Gamma=\sum_{k=0}^{\infty} \hbar^{k} \Gamma_{k}
$$

and substitute it into Eq. (2.19). This yields

$$
\begin{aligned}
& \Theta \Gamma_{1}=0 \\
& \Theta \Gamma_{2}+\int d^{3} x \sum_{\phi} \frac{\delta \Gamma_{1}}{\delta \phi} \frac{\delta \Gamma_{1}}{\delta K_{\phi}}=0, \\
& \Theta \Gamma_{3}+\int d^{3} x \sum_{\phi}\left(\frac{\delta \Gamma_{1}}{\delta \phi} \frac{\delta \Gamma_{2}}{\delta K_{\phi}}+\frac{\delta \Gamma_{2}}{\delta \phi} \frac{\delta \Gamma_{1}}{\delta K_{\phi}}\right)=0,
\end{aligned}
$$

where

$$
\Theta=\int d^{3} x\left[\sum_{\phi}\left(\frac{\delta \Gamma_{0}}{\delta \phi} \frac{\delta}{\delta K_{\phi}}+\frac{\delta \Gamma_{0}}{\delta K_{\phi}} \frac{\delta}{\delta \phi}\right)+b \frac{\delta}{\delta \hat{c}}+i \bar{\zeta} \frac{\delta}{\delta \overline{\hat{\varphi}}}+h \frac{\delta}{\delta \hat{\omega}}\right]
$$

is the Slavnov-Taylor operator. The operator $\Theta$ satisfies $\Theta^{2}=0$ and $[\Theta, \delta]=0$, a property that we will use in Section 5.

We have two different bases of fields and sources. On the one hand, there is the basis formed by $\mathcal{B}_{V A}=\left\{V_{\mu}^{a}, \Lambda^{a}, \chi^{a}, \ldots, K_{V}^{a \mu}, \bar{K}_{\Lambda}^{a}, \bar{K}_{\chi}^{a}, \ldots\right\}$, and on the other hand, there is the basis formed by $\mathcal{B}_{A \lambda}=\left\{A_{\mu}^{a}, \lambda^{a}, \chi^{a}, \ldots, K_{A}^{a \mu}, \bar{K}_{\lambda}^{a}, \bar{K}_{\chi}^{a}, \ldots\right\}$. We are interested in the effective action for the fields and sources in the first basis because that is the effective action which is supersymmetric at tree level. To actually compute it, we could use Feynman rules for the elements of $\mathcal{B}_{V A}$. However, this way to proceed is not convenient, since the Feynman rules for the Yang-Mills and Chern-Simons actions $S_{\mathrm{YM}}$ and $S_{\mathrm{CS}}$ are very complicated in terms of $V_{\mu}^{a}$ and $A^{a}$. Therefore, we will use the Feynman rules 
Table 4

1PI superficially divergent diagrams for $\Gamma_{0}$

\begin{tabular}{|c|c|c|c|c|c|c|c|c|}
\hline \multicolumn{6}{|c|}{ External lines } & \multirow{2}{*}{$\begin{array}{l}1 \text { loop } \\
\bar{\omega}=2\end{array}$} & \multirow{2}{*}{$\begin{array}{l}2 \text { loops } \\
\bar{\omega}=1\end{array}$} & \multirow{2}{*}{$\begin{array}{c}3 \text { loops } \\
\bar{\omega}=0\end{array}$} \\
\hline$x \bar{x}$ & & & & & & & & \\
\hline $\begin{array}{l}\lambda \bar{X} \\
\chi \bar{X} A\end{array}$ & $\begin{array}{l}A^{2} \\
\chi \bar{\chi} H\end{array}$ & $\begin{array}{l}A H \\
(\chi \bar{\chi})^{2}\end{array}$ & $H^{2}$ & & & $\bar{\omega}=1$ & $\bar{\omega}=0$ & \\
\hline $\begin{array}{l}\lambda \bar{\lambda} \\
\chi \bar{\lambda} A \\
(\chi \bar{X})(\chi \bar{\lambda}) \\
(\chi \bar{X})^{2} A\end{array}$ & $\begin{array}{l}\hat{c} c \\
\chi \bar{\lambda} H \\
\chi \bar{\chi} A^{2} \\
(\chi \bar{\chi})^{2} H\end{array}$ & $\begin{array}{l}\hat{\varphi} \bar{\varphi} \\
A^{3} \\
\chi \bar{\chi} A H \\
(\chi \vec{\chi})^{3}\end{array}$ & $\begin{array}{l}\zeta \bar{\chi} \\
A^{2} H \\
\chi \bar{\chi} H^{2}\end{array}$ & $A H^{2}$ & $H^{3}$ & $\vec{\omega}=0$ & & \\
\hline
\end{tabular}

for $A_{\mu}^{a}$ and $\lambda^{a}$, and treat $V_{\mu}^{a}$ and $\Lambda$ as composite fields. Ordinary power counting for $\Gamma_{0}$ shows that the theory is superrenormalizable, with only the 1PI diagrams in Table 4 being superficially divergent. We note that there are no superficially divergent diagrams with external sources as external lines. Let us consider a 1PI diagram with $A_{\mu}^{a}$ and/or $\lambda^{a}$ external lines, and let us denote its superficial UV degree of divergence by $\bar{\omega}$. Then it is straightforward to see that the diagrams that result from replacing one or more of the external $A_{\mu}^{a}$-lines with $f^{a b c} \bar{\chi}^{b} \gamma_{\mu} \chi^{c}$ and one or more of the external $\lambda^{a}$-lines with $f^{a b c} A^{b} \chi^{c}, f^{a b c} H^{b} \chi^{c}$ or $f^{a b c} f^{c d e} \gamma^{\mu} \chi^{b}\left(\bar{\chi}^{d} \gamma_{\mu} \chi^{e}\right)$ all have superficial UV degree of divergence strictly less than $\bar{\omega}$. This is very simple to see for the fields $A_{\mu}^{a}$ and $V_{\mu}^{a}$, since $A_{\mu}^{a}$ couples with a derivative to two other fields $A_{\mu}^{a}$ and to a ghost-antighost pair $\hat{c}^{a} c^{b}$, while $f^{a b c} \bar{\chi}^{b} \gamma_{\mu} \chi^{c}$ only couples to two other fields $\chi^{a}$ without any derivative. For $\lambda^{a}$ and $\Lambda^{a}$, replacing $\lambda$ by $\not \partial \chi^{a}$ does not introduce worse divergences since the couplings with one external $\chi^{a}$ are already taken into account in Table 4. Replacing $\lambda^{a}$ by $f^{a b c} A^{b} \chi^{c}, f^{a b c} H^{b} \chi^{c}$ or $f^{a b c} f^{c d e} \gamma^{\mu} \chi^{b}\left(\bar{\chi}^{d} \gamma_{\mu} \chi^{e}\right)$ requires some more analysis, but as can easily be checked does not lead to worse divergences. All in all, regarding $V_{\mu}^{a}$ and $\Lambda^{a}$ as composite fields does not worsen power counting.

\section{Dimensional regularization, $\epsilon^{\mu \nu \rho}$ and BRS invariance}

\subsection{Dimensional regularization}

Due to the presence of $\epsilon_{\mu \nu \rho}$ in the Chern-Simons action, DReG is not straightforward. To incorporate $\epsilon_{\mu \nu \rho}$ into the framework of DReG, we follow Ref. [3] and use the HVBM prescription for parity-violating objects, originally introduced by 't Hooft and Veltman [2] and systematized by Breitenlohner and Maison [10]. The HVBM prescription defines $\epsilon_{\mu \rho \nu}$ in $n \geqslant 3$ integer dimensions as a completely antisymmetric object in its indices satisfying the relations [10]

$$
\epsilon_{\mu_{1} \mu_{2} \mu_{3}} \epsilon_{\nu_{1} \nu_{2} \nu_{3}}=\sum_{P}(-1)^{|P|} \tilde{g}_{\mu_{1} \nu_{P 1}} \tilde{g}_{\mu_{2} \nu_{P 2}} \tilde{g}_{\mu_{3} \nu_{P 3}}, \quad \epsilon_{\mu_{1} \mu_{2} \mu_{3}} \hat{g}^{\mu_{3} \mu_{4}}=0
$$


where all indices run from 0 to $n-1$, the sum is extended over all permutations $(1,2,3) \rightarrow(P 1, P 2, P 3),|P|$ is the order of the permutation $P, g_{\mu \nu}$ is the metric on $\mathbb{R}^{n}$, and $\tilde{g}_{\mu \nu}$ and $\hat{g}_{\mu \nu}$ are its projections onto $\mathbb{R}^{3}$ and $\mathbb{R}^{n-3}$. In other words, $\epsilon^{\mu \nu \rho}$ is treated as a three-dimensional object. In what follows, we will regard objects with tildes and hats as projections onto $\mathbb{R}^{3}$ and $\mathbb{R}^{n-3}$, respectively. That is, $\tilde{p}_{\mu}=\tilde{g}_{\mu \nu} p^{\nu}, \hat{p}^{2}=\hat{g}_{\mu \nu} p^{\mu} p^{\nu}$, etc. Hatted objects vanish at $n=3$ and are usually called evanescent in the literature. We remark that the HVBM definition of $\epsilon^{\mu \nu \rho}$ outside three dimensions is the only algebraically consistent one known to date [11].

Once we have an algebraically consistent definition for $\epsilon^{\mu \nu \rho}$ in $n$ dimensions, we dimensionally regularize the theory as follows [3]:

(i) First, we extend the Feynman rules from three dimensions to $n$ dimensions.

(ii) Next we construct $n$-dimensional 1PI diagrams and use the techniques of $[2,12]$ to continue $n$ to complex values $d$. This replaces every three-dimensional 1PI diagram in the original theory with a dimensionally regularized diagram defined in terms of dimensionally regularized integrals. It must be emphasized that, when continuing $n$ from integer to complex values, the quantities $p^{2}, p^{\mu}, g_{\mu \nu}$ and $\epsilon^{\mu \nu \rho}$ cease to have meaning as scalars, vectors and tensors and are defined only through their algebraic relations [10].

(iii) Finally, we compute the dimensionally regularized integrals entering in a dimensionally regularized Feynman diagram and analytically continue the result to $d=3$. This defines the value of the dimensionally regularized 1PI diagrams. As usual, computation of dimensionally regularized integrals entails a Wick rotation to euclidean momentum space.

The extension of the Feynman rules from three to $n$ dimensions is obtained as in QCD, except the propagator of the gauge field, which deserves some attention. In three dimensions, the quadratic part of the action in $A_{\mu}^{a}$ and $b^{a}$ has in momentum space the form

$$
-\frac{1}{2} \int \frac{d^{3} p}{(2 \pi)^{3}}\left[A_{\rho}^{a}(p) K^{\rho \mu}(p) A_{\mu}^{a}(-p)+b^{a}(p) p^{\rho} A_{\rho}^{a}(-p)\right],
$$

where

$$
K^{\rho \mu}(p)=\frac{1}{g^{2}}\left[-\epsilon^{\rho \sigma \mu} p_{\sigma}+\frac{i}{m}\left(p^{2} g^{\rho \mu}-p^{\rho} p^{\mu}\right)\right] .
$$

This defines the kinetic matrix of $A_{\mu}^{a}$ and $b^{a}$ as

$$
T(p)=\left(\begin{array}{cc}
K^{\rho \mu}(p) & -p^{\rho} \\
p^{\mu} & 0
\end{array}\right) .
$$

Inverting $T(p)$ in three dimensions, we obtain the three-dimensional propagators for the fields $A_{\mu}^{a}$ and $b^{a}$, namely

$$
\left\langle A_{\mu}^{a}(p) A_{\nu}^{b}(-p)\right\rangle_{0}^{(3)}=\delta^{a b} D_{\mu \nu}(p)
$$

and 


$$
\left\langle A_{\mu}^{a}(p) b^{b}(-p)\right\rangle_{0}^{(3)}=\delta^{a b} \frac{p_{\mu}}{p^{2}}
$$

with $D_{\mu \nu}(p)$ given by

$$
D_{\mu \nu}(p)=-\frac{g^{2} m}{p^{2}\left(p^{2}+m^{2}-i 0\right)}\left(m \epsilon_{\mu \rho \nu} p^{\rho}+i p^{2} g_{\mu \nu}-i p_{\mu} p_{\nu}\right) \text {. }
$$

According to the arguments in Ref. [10], for BRS invariance to be manifestly preserved, the $n$-dimensional propagators for $A_{\mu}^{a}$ and $b^{a}$ should be computed by inverting the kinetic matrix $T(p)$ in $n$ dimensions. Doing this and using for $\epsilon^{\mu \nu \rho}$ the HVBM definition given above, we obtain

$$
\left\langle A_{\mu}^{a}(p) A_{\nu}^{b}(-p)\right\rangle_{0}^{(n)}=\delta^{a b} \Delta_{\mu \nu}(p), \quad\left\langle A_{\mu}^{a}(p) b^{b}(-p)\right\rangle_{0}^{(n)}=\delta^{a b} \frac{p_{\mu}}{p^{2}},
$$

where $\Delta_{\mu \nu}(p)$ has the form

$$
\begin{aligned}
\Delta_{\mu \nu}(p)= & -\frac{g^{2} m}{\left(p^{2}-i 0\right)^{2}+m^{2} \tilde{p}^{2}}\left[m \epsilon_{\mu \rho \nu} p^{\rho}+i p^{2} g_{\mu \nu}-i p_{\mu} p_{\nu}\right. \\
& \left.+\frac{i m^{2}}{p^{2}-i 0}\left(\tilde{p}^{2} \hat{g}_{\mu \nu}+\frac{\hat{p}^{2}}{p^{2}} p_{\mu} p_{\nu}-p_{\mu} \hat{p}_{\nu}-\hat{p}_{\mu} p_{\nu}+\hat{p}_{\mu} \hat{p}_{\nu}\right)\right] .
\end{aligned}
$$

The complicated dependence of $\Delta_{\mu \nu}(p)$ on $\tilde{p}^{\mu}$ and $\hat{p}^{\mu}$ arises from the fact that $\epsilon^{\mu \nu \rho}$ in $n$ dimensions transforms covariantly under $s o(1,2) \times s o(n-3)$ rather than under the full Lorentz group $s o(1, n)$, and is the price to pay for manifest BRS invariance. We emphasize that we want manifest BRS invariance, since our proof of perturbative finiteness in the next section is based on the fact that DReG manifestly preserves BRS invariance.

To avoid a propagator as involved as $\Delta_{\mu \nu}(p)$, one might wish to simply take the expression for $D_{\mu \nu}(p)$ in Eq. (3.4) and regard $\epsilon_{\mu \nu \rho}$ as defined above and $p^{\mu}$ and $g_{\mu \nu}$ as $n$-dimensional. This way to proceed simplifies the calculations but does not manifestly preserve BRS invariance. To see this, let us consider the matrix propagator corresponding to the propagator $D_{\mu \nu}(p)$, i.e.

$$
M_{D}(p)=\left(\begin{array}{cc}
D_{\mu \nu}(p) & \frac{p_{\mu}}{p^{2}} \\
-\frac{p_{\nu}}{p^{2}} & 0
\end{array}\right)
$$

and let us invert it in $n$ dimensions. The result is not the kinetic matrix $T(p)$ but rather

$$
T_{D}(p)=\left(\begin{array}{cc}
K^{\rho \mu}(p)+B^{\rho \mu}(p) & -p^{\rho} \\
p^{\mu} & 0
\end{array}\right),
$$

where

$$
\begin{aligned}
B^{\rho \mu}(p)= & -\frac{1}{g^{2}} \frac{m}{\left(p^{2}-i 0\right)^{2}+m^{2} \tilde{p}^{2}}\left[\hat{p}^{2}\left(m \epsilon^{\rho \sigma \mu} p_{\sigma \sigma}-i p^{2} g^{\rho \mu}+i p^{\rho} p^{\mu}\right)\right. \\
& \left.-i\left(p^{2}+m^{2}\right)\left(\frac{\hat{p}^{2}}{p^{2}} p^{\rho} p^{\mu}+\tilde{p}^{2} \hat{g}^{\rho \mu}-p^{\rho} \hat{p}^{\mu}-\hat{p}^{\rho} p^{\mu}+\hat{p}^{\rho} \hat{p}^{\mu}\right)\right] .
\end{aligned}
$$




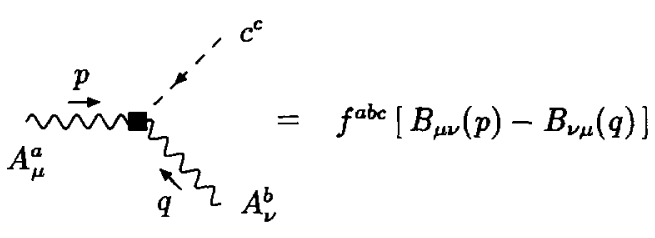

(a)

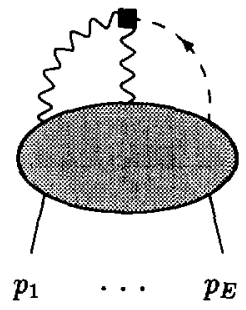

(b)

Fig. 1. The BRS breaking for DReG based on the propagator $\Delta G\left(p_{e}\right)$.

The arguments in Ref. [10] then imply that the BRS identity for a dimensionally regularized 1PI Green function $G\left(p_{e}\right) \equiv G\left(p_{1}, \ldots, p_{E}\right)$ computed with the propagator $D_{\mu \nu}(p)$ contains an extra BRS-violating term which arises because the evanescent contribution

$$
\frac{i}{2} \int d^{n} x d^{n} y A_{\mu}^{a}(x) B^{\mu \nu}(x-y) A_{\nu}^{a}(y)
$$

to the action is not BRS invariant. Indeed, the BRS variation of (3.6) produces an evanescent vertex $O_{\mu \nu}^{a b c}(p, q)=f^{a b c}\left[B_{\mu \nu}(p)-B_{\nu \mu}(q)\right]$ in the dimensionally regularized perturbation series for $G\left(p_{e}\right)$. Formally, the breaking can be written as

$$
\Delta G\left(p_{e}\right)=\lim _{d \rightarrow 3}\left[G\left(p_{e}\right) \int d^{d} p d^{d} q f^{a b c} A_{\mu}^{a}(-p) B^{\mu \nu}(p) A_{\nu}^{b}(q) c^{c}(p-q)\right]_{D},
$$

where $[\ldots]$ denotes $1 \mathrm{PI}$ and the subscript $D$ refers to the propagator $D_{\mu \nu}(p)$. Pictorially, the vertex $O_{\mu \nu}^{a b c}(p, q)$ is depicted in Fig. 1a and the breaking in Fig. 1b. Note that the difference between $\Delta_{\mu \nu}(p)$ and $D_{\mu \nu}(p)$ is again a purely evanescent object, since

$$
\Delta_{\mu \nu}(p)=D_{\mu \nu}(p)+R_{\mu \nu}(p)
$$

and

$$
\begin{aligned}
R_{\mu \nu}(p)= & -\frac{g^{2} m^{3}}{\left(p^{2}-i 0\right)^{2}+m^{2} \tilde{p}^{2}} \\
& \times\left[\frac{\hat{p}^{2}}{p^{2}\left(p^{2}+m^{2}-i 0\right)}\left(m \epsilon_{\mu \rho \nu} p^{\rho}+i p^{2} g_{\mu \nu}+\frac{i m^{2}}{p^{2}-i 0} p_{\mu} p_{\nu}\right)\right. \\
& \left.+\frac{i}{p^{2}-i 0}\left(\tilde{p}^{2} \hat{g}_{\mu \nu}-p_{\mu} \hat{p}_{\nu}-\hat{p}_{\mu} p_{\nu}+\hat{p}_{\mu} \hat{p}_{\nu}\right)\right]
\end{aligned}
$$

vanishes at $n=3$. The identity (3.8) will be used in the next section. It is very important to note that $\Delta_{\mu \nu}(p)$ and $D_{\mu \nu}(p)$ have both UV degree -2 , whereas $R_{\mu \nu}(p)$ has UV degree -4 . As concerns IR power counting, $\Delta_{\mu \nu}(p)$ and $R_{\mu \nu}(p)$ have IR degree -2 , and $D_{\mu \nu}(p)$ has IR degree -1 . 


\subsection{Regularization by dimensional reduction}

Let us very briefly recall the basics of regularization by $\mathrm{DReD}$. In the original formulation of $\mathrm{DReD}$ [4], all the fields and matrices are kept three-dimensional, so that the Dirac algebra of the Feynman diagrams is performed in three dimensions. The momenta however are continued in the sense of ordinary DReG to $d<3$. This way to proceed manifestly preserves supersymmetry since the Dirac algebra is performed in three dimensions and thus Fierz identities remain valid.

The kinetic matrix that results from DReD's prescription is the same as $T(p)$ in Eq. (3.2), where now $\epsilon_{\mu \nu \rho}$ and $g_{\mu \nu}$ are three dimensional and $p^{\mu}$ is $d$-dimensional $(d<3)$. The propagator for the gauge field that results from inverting this matrix has the form in Eq. (3.4), where again $\epsilon_{\mu \nu \rho}$ and $g_{\mu \nu}$ are three dimensional and $p^{\mu}$ is $d$ dimensional $(d<3)$. The fact that the DReD propagator for the gauge field corresponds to the inverse of the kinetic matrix, however, is not enough to secure BRS invariance, since the BRS transformations are not the same as for the unregularized theory. Indeed, whereas the first $d<3$ components of the gauge field have the same BRS transformation law as the gauge field in the unregularized theory, namely $s A_{\mu}^{a}=\left(D_{\mu} c\right)^{a}$, the last $3-d$ components transform as $s A_{\mu}^{a}=f^{a b c} A_{\mu}^{b} c^{c}$. Hence the Ward identity corresponding to this DReD BRS transformation is not the same as the BRS identity for the unregularized theory. In this sense, we say that DReD does not manifestly preserve BRS invariance. It may happen that at the end of all calculations, once the limit $d \rightarrow 3$ has been taken, all effects due to the splitting of the gauge field into $d$ and $3-d$ components go away, but this is not what is meant by manifest BRS invariance. As a matter of fact, in this paper we are going to show that indeed DReD's splitting does not modify the BRS identities when the limit $d \rightarrow 3$ is taken.

As is well known, DReD is algebraically inconsistent because different contractions of three or more $\epsilon^{\mu \nu \rho}$ factors yield different results in $d<3$ dimensions [5]. However, in our model, this inconsistency is absent since the contributions with three or more factors $\epsilon^{\mu \nu \rho}$ are finite by power counting, due to the fact that for large momenta the Yang-Mills action gives the dominant contribution.

\section{Perturbative finiteness}

In what follows we prove that all dimensionally regularized 1PI Green functions of the fields $\psi^{a}$ and the sources $K_{\phi}^{a}$ are finite to all orders in perturbation theory, meaning that no poles arise in them when the regulator $d$ is taken to 3 . Before presenting the proof, let us recall the following property of dimensionally regularized integrals, due to Speer [13]. Consider the dimensionally regularized integral

$$
I_{\mu_{1} \ldots \mu_{N}}\left(p_{e}, m, d\right)=\int \frac{d^{d} q}{(2 \pi)^{d}} \frac{q_{\mu_{1}} \cdots q_{\mu_{N}}}{\prod_{r, s}\left(Q_{r}^{2}\right)^{n_{r}}\left(Q_{s}^{2}+m^{2}\right)^{n_{s}}},
$$


where $Q_{r}^{\mu}$ and $Q_{s}^{\mu}$ are linear combinations of the loop momentum $q^{\mu}$ and the external momenta $p_{e}^{\mu}$, and $n_{r}$ and $n_{s}$ are non-negative integers. Then analytic continuation of $l_{\mu_{1} \ldots \mu_{N}}\left(p_{e}, m, d\right)$ to $d \rightarrow n_{0}$, with $n_{0}$ odd, does not produce poles, even though $I_{\mu_{1} \ldots \mu_{N}}\left(p_{e}, m, d\right)$ might not be finite by power counting at $d=n_{0}$. We call dimensionally regularized integrals of this type Speer integrals. It is important to note that the denominator in the integrand in Eq. (4.1) is Lorentz covariant. Hence, dimensionally regularized integrals with factors in the denominator of the type $p^{4}+m^{2} \tilde{p}^{2}$ arising from internal gauge lines are not of Speer type. Let us now proceed with the proof.

\subsection{One loop}

We recall from Section 2 that superficially divergent one-loop 1PI diagrams for component fields have $\bar{\omega}=0,1,2$. Furthermore, some very simple power counting show that all 1PI one-loop diagrams have IR degree $\underline{\omega} \geqslant 1$. Let us consider a superficially divergent one-loop 1PI diagram and call $\mathcal{D}(d)$ to the corresponding dimensionally regularized diagram. If the diagram does not have internal gauge lines, $\mathcal{D}(d)$ is made of dimensionally regularized integrals of Speer type and hence does not give rise to poles as $d \rightarrow 3$. So we only have to consider superficially divergent diagrams with internal gauge lines. We distinguish two cases: $\bar{\omega}=2$ and $\bar{\omega}=0,1$.

Case $\bar{\omega}=2$. The only one-loop 1PI diagrams with $\bar{\omega}=2$ are the $\chi^{a} \bar{\chi}^{b}$ self-energy graphs. It is easy to see from Eq. (2.14) that there are no such graphs with internal gauge lines.

Case $\bar{\omega}=0,1$. Using for the propagator of each gauge line the decomposition in Eq. (3.8), we write $\mathcal{D}(d)$ as the sum of two contributions: $\mathcal{D}(d)=\mathcal{D}_{D}(d)+\mathcal{D}_{R}(d)$. The first one, $\mathcal{D}_{D}(d)$, arises from replacing every propagator $\Delta_{\mu \nu}(p)$ with $D_{\mu \nu}(p)$ and is of Speer type. The second one, $\mathcal{D}_{R}(d)$, contains contributions with one or more $R_{\mu \nu}$ and is not of Speer type. Since the original diagram had $\bar{\omega} \leqslant 1$ and $\underline{\omega} \geqslant 1$, and every $R_{\mu \nu}$ decreases $\bar{\omega}$ by two units and leaves $\underline{\omega}$ unchanged, $\mathcal{D}_{R}$ is made of dimensionally regularized integrals which are finite at $d=3$ and which are at least linear in $\hat{g}_{\mu \nu}$ :

$$
\hat{g}_{\mu_{1} \nu_{1}} \ldots \hat{g}_{\mu_{N} \nu_{N}} \int d^{d} q \frac{q^{\nu_{1}} \ldots q^{\nu_{N}}}{\prod_{r, s, t}\left(Q_{r}^{2}\right)^{n_{r}}\left(Q_{s}^{2}+m^{2}\right)^{n_{s}}\left(Q_{t}^{4}+m^{2} \tilde{Q}_{t}^{2}\right)^{n_{t}}}, \quad N \geqslant 1 .
$$

Integrals of this type vanish as $d \rightarrow 3$ [12]. Thus $\mathcal{D}_{R}(d) \rightarrow 0$ as $d \rightarrow 3$ and in this limit we are left only with the Speer-type contribution $\mathcal{D}_{D}(d)$, which does not generate poles.

To prove one-loop finiteness, it would have been enough to consider DReD instead of $\mathrm{DReG}$ and use that all one-loop dimensionally regularized integrals arising from $\mathrm{DReD}$ are of Speer type, hence free of poles as $d \rightarrow 3$. This would have avoided the discussion on evanescent contributions. However, DReD and supersymmetry do not by themselves imply finiteness at higher loops, whereas DReG and BRS invariance do (see below).

Note that we have not only proved one-loop finiteness but also that to compute the limit of physical interest $d \rightarrow 3$ we can replace the propagator $\Delta_{\mu \nu}(p)$ with the propagator $D_{\mu \nu}(p)$. Furthermore, since $D_{\mu \nu}(p)$ generates Speer integrals and these do 
not give rise to poles as $d \rightarrow 3$, we can equally well perform the Lorentz algebra of the Feynman diagrams directly in three dimensions. So, all in all, DReG and DReD give the same one-loop Green functions.

\subsection{Higher loops}

At two loops we proceed differently since Speer's result only holds at one loop. Let us assume that there are divergences at two loops when $d \rightarrow 3$. Then the two-loop correction $\Gamma_{2}^{\mathrm{DReG}}$ to the effective action will consist in the limit $d \rightarrow 3$ of a divergent part $\Gamma_{2 \text {,div }}^{\mathrm{DReG}}$ and a finite part $\Gamma_{2, \text { fin }}^{\mathrm{DReG}}$. Since $\Gamma_{2}^{\mathrm{DReG}}$ satisfies the BRS identity $(2.21)$ and $\Gamma_{1}^{\mathrm{DReG}}$ is finite, the divergent part $\Gamma_{2 \text {,div }}^{\mathrm{DReG}}$ satisfies the equation $\Theta \Gamma_{2 \text {,div }}^{\mathrm{DReG}}=0$. Because $1 \mathrm{PI}$ Feynman diagrams involving external sources are finite by power counting and there are no one-loop subdivergences, $\Gamma_{2 \text {,div }}^{\mathrm{DReG}}$ does not depend on the external sources and $\Theta \Gamma_{2 \text {,div }}^{\mathrm{DReG}}=0$ reduces to $s \Gamma_{2 \text {,div }}^{\mathrm{DReG}}=0$, with $s$ the BRS operator. We recall from Table 4 that all two-loop superficially divergent 1PI diagrams with $A_{\mu}^{a}$ and $\lambda^{a}$ external lines have $\bar{\omega}=0$. Hence the most general form of $\Gamma_{2 \text {,div }}^{\mathrm{DReG}}$ compatible with power counting is

$$
\Gamma_{2, \mathrm{div}}^{\mathrm{DReG}}=\frac{1}{d-3} P_{\bar{\omega}_{2}},
$$

where $P_{\bar{\omega}_{2}}$ is given by

$$
\begin{aligned}
P_{\bar{\omega}_{2}}= & m \int d^{3} x\left[\alpha_{1} m \bar{\chi}^{a} \chi^{a}+\alpha_{2} \bar{\chi}^{a} \not \chi^{a}+\alpha_{3} \bar{\chi}^{a} \lambda^{a}+\alpha_{4} A^{a} A^{a}+\alpha_{5} H^{a} H^{a}\right. \\
& \left.+\alpha_{6} f^{a b c} \bar{\chi}^{a} A^{b} \chi^{c}+\alpha_{7} f^{a b c} f^{c d e}\left(\bar{\chi}^{a} \gamma^{\mu} \chi^{b}\right)\left(\bar{\chi}^{d} \gamma_{\mu} \chi^{e}\right)\right]
\end{aligned}
$$

and $\alpha_{1}, \ldots, \alpha_{7}$ are numerical coefficients. In writing the expression for $\Gamma_{2, \mathrm{div}}^{\mathrm{DReG}}$ we have used that two-loop contributions to 1PI Green functions arising from evanescent operators $R_{\mu \nu}(p)$ are finite by power counting and therefore free of poles. The terms in $P_{\bar{\omega}_{2}}$ correspond to all Lorentz invariant two-loop divergences that can be constructed from Table 4 with $\bar{\omega}_{2}$ derivatives. The equation $s \Gamma_{2 \text {,div }}^{\mathrm{DReG}}=0$ is an equation in the coefficients $\alpha_{i}$, whose only solution is $\alpha_{i}=0$. This implies $\Gamma_{2 \text {,div }}^{\mathrm{DReG}}=0$ and proves finiteness at two loops.

The proof at three loops is analogous. In this case, the would-be three-loop divergent contribution $\Gamma_{3 \text {,div }}^{\mathrm{DReG}}$ to the effective action has the form

$$
\Gamma_{3, \mathrm{div}}^{\mathrm{DReG}}=\frac{\alpha m^{2}}{d-3} \int d^{3} x \bar{\chi}^{a} \chi^{a}
$$

and satisfies the equation $\Theta \Gamma_{3 \text {,div }}^{\mathrm{DReG}}=0$, whose only solution is $\Gamma_{3 \text {,div }}^{\mathrm{DReG}}=0$. At higher loops finiteness is trivial, since there are no subdivergences and all 1PI are superficially convergent. Since all 1PI Green functions for the elementary fields are finite to all orders in perturbation theory, we conclude that the beta functions of $g$ and $m$ and the anomalous dimensions of the elementary fields vanish to all orders in perturbation theory.

Let us finally see why $\mathrm{DReD}$ and supersymmetry do not imply finiteness at two, hence at higher loops. Suppose we use DReD, instead of DReG. Since it preserves 
supersymmetry, the divergent part $\Gamma_{2 \text {,div }}^{\mathrm{DReD}}$ of the resulting two-loop effective action must be supersymmetric. In other words, it should have the form $\Gamma_{2 \text {,div }}^{\mathrm{DReD}}=\frac{1}{d-3} P_{\bar{\omega}_{2}}^{\text {susy }}$, with $P_{\bar{\omega}_{2}}^{\text {susy }}$ a supersymmetry invariant. From $P_{\bar{\omega}_{2}}$ in Eq. (4.3) above one can construct the supersymmetry invariant

$$
\begin{aligned}
P_{\bar{\omega}_{2}}^{\text {susy }}= & \alpha m \int d^{3} x\left[\frac{1}{2} \bar{\chi}^{a} \not \partial \chi^{a}+\bar{\chi}^{a} \lambda^{a}+A^{a} A^{a}-H^{a} H^{a}\right. \\
& \left.-\frac{1}{48} f^{a b c} f^{c d e}\left(\bar{\chi}^{a} \gamma^{\mu} \chi^{b}\right)\left(\bar{\chi}^{d} \gamma_{\mu} \chi^{e}\right)\right],
\end{aligned}
$$

with $\alpha$ an arbitrary numerical coefficient. Hence, supersymmetry by itself does not prove finiteness. This is why we have used DReG and BRS invariance.

\section{The effective action}

Since the theory is finite, every regularization method defines a renormalization scheme. Let us consider the following two renormalization schemes: scheme $\mathcal{R}^{\mathrm{DReG}}$ uses as regulator DReG and performs no subtractions, and scheme $\mathcal{R}^{\mathrm{DReD}}$ uses $\mathrm{DReD}$ and performs no subtractions. We want to prove that the difference $\Delta \Gamma=\Gamma^{\mathrm{DReG}}-\Gamma^{\mathrm{DReD}}$ between the corresponding effective actions is zero. We have already seen in Section 4 that this is indeed the case at one loop. So let us consider the two-loop case.

According to general results from renormalization theory $[6,10]$, the difference $\Delta \Gamma_{2}$ at two loops can at most have the form (1.2), with $P_{\bar{\omega}_{2}}$ as in Eq. (4.3). Since DReG preserves BRS invariance manifestly, $\Gamma_{2}^{\mathrm{DReG}}$ satisfies the BRS identity at two loops (2.21). Substituting Eq. (1.2) in Eq. (2.21), acting from the left with the supersymmetry generator $\delta$ and using $\Gamma_{1}^{\mathrm{DReG}}=\Gamma_{1}^{\mathrm{DReD}}$ and $[\Theta, \delta]=0$, we obtain $\Theta \delta P_{\bar{\omega}_{2}}=0$. Since $P_{\bar{\omega}_{2}}$ does not depend on external sources and $\delta$ acting on the components of the gauge multiplet does not produce external sources, $\delta P_{\bar{\omega}_{2}}$ is independent of external sources. Therefore the equation $\Theta \delta P_{\bar{\omega}_{2}}=0$ reduces to $s \delta P_{\bar{\omega}_{2}}=0$, which is an equation for the coefficients $\alpha_{i}, s$ being the BRS operator. Since $\delta P_{\bar{\omega}_{2}}$ depends polynomially on the components of the gauge multiplet and their derivatives and has an overall factor of $m$, any non-trivial $\delta P_{\bar{\omega}_{2}}$ satisfying $s \delta P_{\bar{\omega}_{2}}=0$ should be $m$ times a BRS invariant of mass dimension two. However, there are no such invariants. Hence, $\delta P_{\bar{\omega}_{2}}=0$. The only supersymmetry invariant that can be formed from $P_{\bar{\omega}_{2}}$ is $P_{\bar{\omega}_{2}}^{\text {susy }}$, which in terms of superfields takes the form in Eq. (1.4). At this point we have exhausted all information given by BRS symmetry and supersymmetry. The only way left to determine the value of the coefficient $\alpha$ in $P_{\bar{\omega}_{2}}^{\text {susy }}$ is to compute it using Feynman diagrams. We do this below and find that $\alpha=0$.

At three loops, the difference $\Delta \Gamma_{3}$ is

$$
\Delta \Gamma_{3}=\alpha m^{2} \int d^{3} x \bar{\chi}^{a} \chi^{a} .
$$

Since $\Delta \Gamma_{3}$ is not BRS invariant, nor supersymmetric, the same arguments as used at the two-loop level are now powerful enough to conclude that $\alpha=0$ without the need of any 


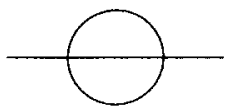

(a)

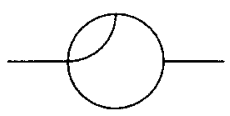

(d)

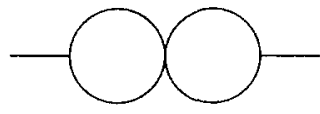

(b)

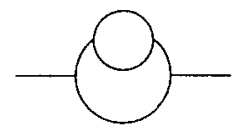

(e)

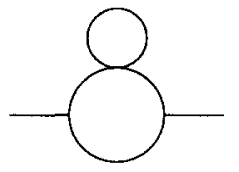

(c)

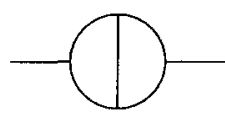

(f)

Fig. 2. Two-loop 1PI topologies for the proper Green function $H H$.

explicit computation. At higher loops, the difference $\Delta \Gamma$ vanishes since at one, two and three loops it vanishes and there are no overall divergences by power counting.

We are left with the computation of the coefficient $\alpha$. To calculate it, it is enough to evaluate the difference between the contributions from DReG and DReD to one of the five proper functions in Eq. (4.4). The simplest case to compute is the self-energy of the field $H^{a}$. The vertices with an $H$ are

$$
H \zeta \chi, \quad H \hat{\varphi} \varphi, \quad H \hat{\omega} c, \quad H \hat{\varphi} \chi c
$$

Using these vertices, one can construct two-loop 1PI diagrams with the six topologies in Fig. 2. In fact, no graphs with the topology of Fig. 2a can be constructed, since there is no four-point vertex containing the fields $H, \varphi$ and $\hat{c}$ (note that $\hat{\varphi}$ only propagates in $\varphi$ and $c$ into $\hat{c}$.) The topologies in Figs. $2 b$ and $2 c$, being products of one-loop topologies, give the same contributions in $\mathrm{DReG}$ as in $\mathrm{DReD}$, hence they do not contribute to $\alpha$. We are thus left with the topologies in Figs. $2 \mathrm{~d}-2 \mathrm{f}$.

Since one-loop subdiagrams give the same contributions in DReG as in DReD, we only need to consider the overall divergent parts of the two-loop diagrams. Since the diagrams are only logarithmically divergent by power counting, we may set the external momentum $p^{\mu}$ and the mass $m$ equal to zero in the numerators (except, of course, of the overall factor $m$ ). Because every epsilon in the propagator and in the three-vertex of the gauge field $A_{\mu}^{a}$ reduces the overall degree of divergence by one unit, the overall divergent part of every diagram is epsilon-independent. All this gives for the overall divergent part of every diagram an expression of the form

$$
\int \frac{d^{d} k}{(2 \pi)^{d}} \frac{d^{d} q}{(2 \pi)^{d}} \frac{N(k, q)}{D(k, q, p, m)} .
$$

The numerator $N(k, q)$ always contains a trace over a fermion loop. This is obvious for those diagrams in which $H$ couples to fermions. The only vertex where $H$ does not couple to fermions is the vertex $H \hat{\omega} c$, but in this case $\hat{\omega}$ propagates into $\omega$ and now $\omega$ couples to fermions. In fact, no two-loop diagram with this structure can be constructed. As far as the diagrams with internal gauge lines are concerned, they only occur in 
topology $4 \mathrm{e}$ and closer inspection reveals that their contributions separately cancel. Anyhow, even if they had not cancelled, one could have decomposed the propagator $\Delta_{\mu \nu}(p)$ into a covariant part $D_{\mu \nu}(p)$ and an evanescent part $R_{\mu \nu}(p)$. The latter part yields an evanescent contribution which is finite by power counting and hence vanishes. Thus the contributions of both $\mathrm{DReG}$ and $\mathrm{DReD}$ are the same except for the trace over the fermions. The trace of a sum of products of $\phi$ and $k$ can always be written as $d$-dimensional scalar products $k^{2}, k q$ and $q^{2}$ times an overall trace of the unit matrix. Since this trace is different in DReG and DReD, after summing over diagrams, $\alpha$ can be written as

$$
\alpha=\left(\operatorname{tr}_{\mathrm{DReG}} 1-\operatorname{tr}_{\mathrm{DReD}} 1\right) \int \frac{d^{d} k}{(2 \pi)^{d}} \frac{d^{d} q}{(2 \pi)^{d}} \frac{f\left(k^{2}, k q, q^{2}\right)}{D_{T}(k, q, p, m)},
$$

where $f\left(k^{2}, k q, q^{2}\right)$ is a polynomial of its arguments. Since we have already shown that the theory is finite, the integral is finite and therefore the difference due to the trace vanishes in the limit $d \rightarrow 3$. Hence $\alpha=0$.

\section{Further comments}

We conclude with a few comments.

(1) The equality of the two effective actions considered in this paper is not explained by the standard theorems of renormalizable quantum field theory. One possible explanation might be that there exists a third, as yet unknown, symmetry of the model. Another explanation might be that the existing theorems of local quantum field theory $[6,7]$ concerning the difference between two renormalized expressions for the same Green function computed in two different renormalization schemes can be sharpened for finite models which are superrenormalizable by power counting and which have symmetries.

(2) For the purely bosonic theory, it has been claimed without proof that since the theory is superrenormalizable, subtleties due to the epsilon tensor should not matter [14]. This can easily be proved for both the bosonic and the supersymmetric theories. Clearly, such subtleties may only arise from superficially divergent graphs. At one loop, since there are no 1PI graphs with $\bar{\omega}=2$ containing internal gauge lines nor three-gauge vertices, and since every epsilon occurring in a IPI graph decreases the UV superficial degree of divergence of the graph by one unit, only 1PI graphs with $\bar{\omega}=1$ may produce epsilon ambiguities. The ambiguities, if any, will be linear in $\epsilon^{\mu \nu \rho}$ and independent of the external momenta, since they must depend polynomially on the external momenta and arise from Feynman integrals that are logarithmically divergent. It is very easy to see that, out of just one $\epsilon^{\mu \nu \rho}$ and nothing else, no Lorentz invariant can be constructed for the 1PI Green functions in Table 4 that have $\bar{\omega}=1$ at one loop. Hence, there are no epsilon ambiguities at one loop. The same arguments show that this is also the case at two and higher loops. The source of different results for different regularization methods is not actually the epsilon, but the parity-even sector of the theory. 
(3) In this article we have considered DReG and DReD, but one can also consider a covariantized $\mathrm{DReG}$ method based on the naively covariantized $n$-dimensional propagator $D_{\mu \nu}(p)$ in Eq. (3.4). For the purely bosonic theory it has been shown that this 'covariantized' DReG gives the same effective action as DReG [3]. A straightforward generalization of the arguments given there shows that this is also the case for the supersymmetric case we have considered here.

(4) Our analysis relies on the fact that our three-dimensional model is superrenormalizable by power counting and finite. There exist several one, two [15] and all-loop [16] finite supersymmetric models in four dimensions, and $N=4$ Yang-Mills theory is also finite to all loop orders. It would be interesting to apply the methods developed in this paper to these models. A hint that also for these models under certain conditions DReG and DReD could give the same results is provided by the one-loop analysis of $N=1$ supersymmetric Yang-Mills theory in four dimensions in Ref. [17]. Note, however, that in this reference a non-supersymmetric gauge was used.

(5) In addition to Yang-Mills-Chern-Simons models, there exist Einstein-ChernSimons models (topologically massive gravity) [18,19]. Perhaps our methods can be applied in these cases [20].

(6) Our analysis used component graphs and not supergraphs because we needed $\mathrm{DReG}$ to prove finiteness and DReG cannot be formulated for superfields. In fact, since the classical Yang-Mills and Chern-Simons actions contain many terms when written in terms of the spinor connection $\Gamma_{a}^{a}$ [see Eqs. (2.2, (2.3)], using supergraphs is not that advantageous. Also note that there are no non-renormalization theorems in three dimensions because there are no chiral superfields.

(7) Chern-Simons theory by itself has the problem that there exists no propagator in $n \geqslant 3$ dimensions for the gauge field, even for a non-vanishing gauge-fixing parameter [21]. Hence, for this model, DReG cannot be formulated in a manifestly BRS invariant way. One way to overcome this is to add to the Chern-Simons action the Yang-Mills term [3,21] or any other gauge-invariant parity-even term [22]. Conversely, starting with Yang-Mills theory, one encounters IR divergences on shell. A way to regularize these divergences is to add a Chern-Simons term to the Yang-Mills action [23]. Hence in both cases we end up with Yang-Mills-Chern-Simons theory.

(8) There exist other studies in the literature concerning finiteness of pure ChernSimons theory [24]. They use a particular symmetry of the gauge-fixed action in the Landau gauge called 'vector supersymmetry', which has nothing to do with the ordinary supersymmetry we have discussed. These articles use abstract cohomology arguments and do not discuss regularization, nor they include in the classical action a Yang-Mills term. A cohomological study of $N=2$ Yang-Mills-Chern-Simons theory in a nonsupersymmetric gauge has been performed in Ref. [25], where renormalizability is proven and where, exploiting the fact that the theory has extended supersymmetry, it is shown that radiative corrections to $m$ are finite, making no statement about those to $g$. 


\section{Acknowledgment}

We thank P. Breitenlohner, J.W. van Holten, F. De Jonghe and D. Maison for discussions.

\section{References}

[1] For a superspace approach, see O. Piguet and K. Sibold, Renormalized supersymmetry - The perturbation theory of $N=1$ supersymmetric theories in flat space-time (Birkhauser, Boston, 1986).

For theories in the Wess-Zumino gauge, without auxiliary fields, see P.L. White, Class. Quan. Grav. 9 (1992) 413; N. Maggiore, Int. J. Mod. Phys. A 10 (1995) 3781, 3937; N. Maggiore, O. Piguet and S. Wolf, Nucl. Phys. B 458 (1996) 403; Nucl. Phys. B 476 (1996) 329.

12| G. 't Hooft and M. Veltman, Nucl Phys. B 44 (1972) 189.

\{3| G. Giavarini, C.P. Martin and F. Ruiz Ruiz, Nucl. Phys. B 381 (1992) 222.

|4| W. Siegel, Phys. Lett. 84B (1979) 193.

[5] W. Siegel, Phys. Lett. 94B (1980) 37.

[6] K. Hepp, Renormalization theory, in Statistical Mechanics and quantum field theory, ed. C. DeWitt and R. Stora (Gordon and Breach, New York, 1971).

H. Epstein and V. Glasser, Ann. Inst. Henri Poincaré XIX (1973) 211.

17| D. Maison, Renormalization theory, a short account of results and problems, in Renormalization of quantum field theories with non-linear field transformations, ed. P. Breitenlohner, D. Maison and K. Sibold (Springer, Berlin, 1988)

[8] S.J. Gates Jr., M.T. Grisaru, M. Roček and W. Siegel, Superspace or one thousand and one lessons in supersymmetry (Benjamin, Reading, 1983). W. Siegel, Nucl. Phys. B 156 (1979) 135.

19| J. Zinn-Justin, Renormalization of gauge theories, in Trends in elementary particle physics, Lectures Notes in Physics 37, ed. H. Rollnik and K. Dietz (Springer, Heidelberg, 1975).

$[10]$ P. Breitenlohner and D. Maison, Commun. Math. Phys. 52 (1977) 11.

[11] G. Bonneau, Int. J. Mod. Phys. A (1989).

M. Bos, Ann. Phys. 181 (1988) 197.

H. Osborn, Ann. Phys. 200 (1990) 1.

[12] J.C. Collins, Renormalization (Cambridge University Press, Cambridge, 1987).

[13] E.R. Speer, J. Math. Phys. 15 (1974) 1; Ann. Inst. Henri Poincaré XXII (1975) 1.

114] R. Pisarski and S. Rao, Phys. Rev. D 32 (1985) 2081.

|15] A.J. Parkes and P.C. West, Phys. Lett. B 138 (1984) 99; Nucl. Phys. B 256 (1985) 340.

D.R.T. Jones and L. Mezincescu, Phys. Lett. B 138 (1984) 293.

A.J. Parkes, Phys. Lett. B 156 (1985) 73.

D.R.T. Jones and A.J. Parkes, Phys. Lett. B 160 (1985) 267.

[16] A.V. Ermushev, D.I. Kazakov and O.V. Tarasov, Nucl. Phys. B 281 (1987) 72.

D.I. Kazakov, Phys. Lett. B 179 (1986)952.

C. Lucchesi, O. Piguet and K. Sibold, Helv. Phys. Acta 61 (1988) 321; Phys. Lett. B 201 (1988) 241.

C. Lucchesi, Finiteness in $N=1$ SYM theories, hep-ph $/ 9607368$.

[17] D.M. Capper, D.R.T. Jones and P. van Nieuwenhuizen, Nucl. Phys. B 167 (1980) 479.

[18] S. Deser, R. Jackiw and S. Templeton, Ann. Phys. 140 (1982) 372.

[19] A. Lerda and P. van Nieuwenhuizen, Phys. Rev. Lett. 62 (1989) 1217.

[20] S. Deser and Z. Yang, Class. Quan. Grav, 7 (1990) 1603. B. Keszthelyi and G. Kleppe, Phys. Lett. B 281 (1992) 33.

|21] C.P. Martin Phys. Lett. B 241 (1990) 513.

[22] G. Giavarini, C.P. Martin and F. Ruiz Ruiz, Phys. Rev. D 47 (1993) 5536; Phys. Lett. B 314 (1993) 328; Phys. Lett. B 332 (1994) 345.

[23] J. Schonfeld, Nucl. Phys. B 185 (1981) 157.

R. Jackiw and S. Templeton, Phys. Rev. D 23 (1981) 2291.

[24] A. Blasi and R. Collina, Nucl. Phys. B 345 (1990) 472.

F. Delduc, C. Lucchesi, O. Piguet and S.P. Sorella, Nucl. Phys. B 346 (1990) 313.

C. Lucchesi and O. Piguet, Nucl. Phys. B 381 (1992) 281.

[25] N. Maggiore, O. Piguet and M. Ribordy, Helv. Phys. Acta 68 (1995) 264. 